\title{
Landmark Discrimination Learning in the Dog
}

\author{
Norton W. Milgram, ${ }^{1,5}$ Beth Adams, ${ }^{2}$ Heather Callahan, ${ }^{3}$ Elizabeth Head,${ }^{4}$ \\ Bill Mackay, ${ }^{3}$ Celeste Thirlwell, ${ }^{1}$ and Carl W. Cotman ${ }^{4}$ \\ ${ }^{1}$ Division of Life Sciences \\ University of Toronto at Scarborough \\ Scarborough, Ontario, M1C 1A4 Canada \\ Departments of ${ }^{2}$ Pharmacology and ${ }^{3}$ Physiology \\ University of Toronto, Toronto, Ontario, M5S 1AH Canada \\ ${ }^{4}$ Institute for Brain Aging and Dementia \\ University of California \\ Irvine, California 92717-4550 USA
}

\section{Abstract}

\begin{abstract}
Allocentric spatial memory was studied in dogs of varying ages and sources using a landmark discrimination task. The primary goal of this study was to develop a protocol to test landmark discrimination learning in the dog. Using a modified version of a landmark test developed for use in monkeys, we successfully trained dogs to make a spatial discrimination on the basis of the position of a visual landmark relative to two identical discriminanda. Task performance decreased, however, as the distance between the landmark and the "discriminandum" was increased. A subgroup of these dogs was also tested on a delayed nonmatching to position spatial memory task (DNMP), which relies on egocentric spatial cues. These findings suggest that dogs can acquire both allocentric and egocentric spatial tasks. These data provide a useful tool for evaluating the ability of canines to use allocentric cues in spatial learning.
\end{abstract}

Spatial learning, the ability to locate the position of an object in space, can be achieved in two ways: (1) by reference to the observer's body position (egocentric cues); or (2) by reference to the position of an external referent or landmark (allocentric cues) (Pohl 1973). Previous research has

${ }^{5}$ Corresponding author. demonstrated that dogs can use both visual and auditory cues to solve a spatial delayed response task (Hunter 1913; Lawicka and Konorski 1959). We have extended this work to show that dogs can acquire visuospatial tasks using a nonmatching paradigm (e.g., a spatial version of a delayed nonmatching to position task) (Head et al. 1995). To solve both the delayed response and the delayed nonmatching to position (DNMP) tasks, dogs are very likely to rely on egocentric cues. In both of these tasks, the use of allocentric cues would likely be ineffective because the functional relevance of the available allocentric cues change from trial to trial. To date, allocentric spatial learning has been demonstrated in rats (e.g., Gleason and Rothblat 1994) and monkeys (e.g., Pohl 1973) but not in dogs. Although spatial learning and memory appears to be a phenomenon that is well-conserved among species, it is likely to be particularly important for dogs because they are territorial and it is therefore essential that they be able to learn and to remember the location of objects in space (Gallistel 1990).

A landmark discrimination task has been used to study allocentric spatial learning in monkeys (e.g., Pohl 1973; Ungerleider and Brody 1977; Mishkin et al. 1982; Mishkin and Ungerleider 1982). This task requires that a spatial discrimination be made on the basis of the position of a visual object. In a testing box (Wisconsin General Testing Apparatus; WGTA), monkeys are presented with two identical "discriminanda" objects, which are distinguishable only by their proximity to a landmark object. Animals are rewarded for approaching and displacing the object closest to the land-

LEARNING \& MEMORY 6:54-61 @ 1999 by Cold Spring Harbor Laboratory Press ISSN1072-0502/99 \$5.00

$$
\begin{array}{llllllllllllllll}
L & E & A & R & N & I & N & G & \boldsymbol{\bigotimes} \\
\mathbf{5 4} & M & E & M & O & R & Y
\end{array}
$$


mark object. Solving this task relies solely on the use of allocentric spatial cues because information about the correct response is provided only by the location of the landmark. Egocentric spatial cues, on the other hand, cannot be used to solve the task because the position of the subject per se is irrelevant.

Pohl (1973) further demonstrated that lesions to the posterior parietal cortex, but not the frontal cortex, impaired performance on the landmark task. In contrast, lesions to the frontal cortex, but not the posterior parietal cortex, impair performance on a place discrimination task that relies on egocentric cues (Pohl 1973; Ungerleider and Brody 1977). Whereas these data suggest that dual neural systems associated with the posterior parietal and frontal cortex may mediate allocentric and egocentric spatial learning, respectively, the actual circuitry is undoubtedly more complicated. For instance, the posterior parietal cortex has also been associated with egocentric spatial knowledge (Stein 1989) and imaging studies using positron emission tomography have shown that the parahippocampal region is also associated with allocentric spatial knowledge (e.g., Maguire et al. 1996, 1998).

This study was designed to develop a landmark discrimination learning task for use in the canine to determine whether dogs could use allocentric cues to solve a spatial task.

\section{Materials and Methods}

\section{SUBJECTS}

Nine, random-source, mixed-breed dogs were used: five young males (1.5-2 years; Angus, Benz, Buick, Bronco, and Quincy); two middle-aged males ( $7-8$ years; Santa and Nickie); one old female and one old male ( $>10$ years; Millie and Inu). All dogs had received a standard training protocol before this study to familiarize them with the testing apparatus and the testing procedures (Milgram et al. 1994). This included reward-approach learning, object-approach learning, and object discrimination and reversal learning. In addition, all dogs (except Santa) had been trained previously on a DNMP task before receiving landmark discrimination training.

Dogs were housed individually in $1.07 \times 1.22$ $\mathrm{m}$ pens with continual access to fresh water. Animals were maintained on a 12:12-hr light-dark cycle, the humidity was kept at $40 \%-60 \%$, and the temperature was maintained between $22-24^{\circ} \mathrm{C}$.
Dogs were exercised once daily in the early morning for $\sim 15$ min while their pens were cleaned, and they were fed $\sim 300$ grams of dry dog food once daily between 2:30 and 3:30 p.m. All behavioral testing was conducted in the morning and early afternoon before feeding.

\section{TESTING APPARATUS}

As described previously (Milgram et al. 1994), the test apparatus was a $0.609 \times 1.15 \times 1.08-\mathrm{m}$ wooden box that was based on a canine adaptation of the WGTA described by Fox (1971). The box was equipped with a sliding Plexiglas food tray with two lateral wells and a medial food well. Vertical stainless-steel bars cover the front of the box. The height of each bar was adjustable, so that the size of the opening to each food well could be uniquely set for each dog. The experimenter was separated visually from the dog by a screen with a one-way mirror and a hinged-door on the bottom. Testing occurred in darkness, except for a light with a $60-\mathrm{W}$ bulb that was attached to the front of the box. The hinged door was opened for the presentation and removal of the food tray.

\section{GENERAL TEST PROCEDURES}

Dogs received 10 trials per day with an intertrial interval of $90 \mathrm{sec}$. Dogs were tested once a day until they reached a two-stage criterion. They had to first respond correctly on 9 of 10 trials or on 8 of 10 trials over 2 consecutive days. They then had to respond correctly on at least $70 \%$ of the next 30 trials (over three consecutive sessions). Dogs were tested for a maximum of 40 days (for a total of 400 trials) or until they reached criterion, whichever came first.

\section{DATA ACQUISITION}

Data acquisition was controlled using a customized computer program that controlled all timing and randomization procedures, indicated the location of the reward and the appropriate stimulus objects, and stored all of the data in data files. Before the beginning of each trial, the computer emitted a tone that served as a cue for the dog and instructed the experimenter to deliver the food tray. Each trial was started when the experimenter pressed a key and simultaneously presented the tray to the subject. The dogs' responses were re- 
corded by a key press, which also indicated the end of the trial and signaled the beginning of the intertrial interval.

\section{Results}

Experiment 1: Development of a Paradigm for Training Dogs on a Landmark Discrimination Learning Task

Experiment 1 was aimed at developing a protocol for training dogs on a landmark discrimination task based on the procedure used by Pohl (1973). We initially attempted to train dogs on a task that required them to associate a food reward with the discriminandum closest to the landmark.

\section{PROTOCOL 1}

Two yellow, rectangular Lego blocks $(7 \times 6 \times 6 \mathrm{~cm})$ were used as identical discriminanda and a thin rectangular yellow block of wood $(9 \times 2 \times 2 \mathrm{~cm})$ was used as the landmark object. The yellow Lego blocks were placed $23 \mathrm{~cm}$ apart to cover the two lateral food wells on the presentation tray. The middle food well was not used in this study. The landmark object was placed diagonally and medially $1 \mathrm{~cm}$ away from the discriminandum that covered the reward (Fig. 1). The landmark was attached to the food tray with a piece of

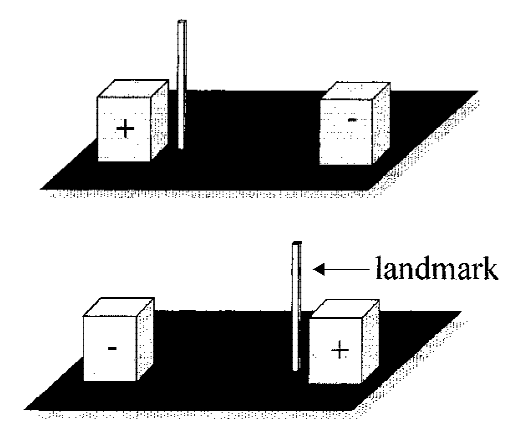

Figure 1: Landmark and discriminanda configuration for Protocol 1 in Experiment 1. Two rectangular Lego blocks were used as identical discriminanda and a thin rectangular block of wood was used as the landmark object. The Lego blocks were placed $23 \mathrm{~cm}$ apart to cover the two lateral food wells on the presentation tray. The landmark object was placed diagonally and medially $1 \mathrm{~cm}$ away from the discriminandum that covered the reward.
Velcro. The "correct" (i.e., rewarded) side was determined randomly by the computer, with the constraint that both sides were correct on half of the trials each test session. On each trial, the experimenter placed the food reward under either the left or right food well and positioned the landmark accordingly. The screen was then raised, the tray was presented to the dog, and the dog was allowed to respond to one side. A single correction trial was allowed after the first incorrect response.

After 400 trials, none of the dogs had reached the preset criterion of nine correct responses out of 10 responses or eight correct responses out of 10 responses on 2 consecutive days. The dogs' failure to learn this task was surprising because monkeys (Pohl 1973) and rats (Gleason and Rothblat 1994; Biegler and Morris 1996) can acquire similar landmark tasks using comparable protocols. It is possible, however, that the dogs did not associate the reward with the discriminandum that was closest to the landmark because the distance (i.e., 1 $\mathrm{cm})$ between the landmark and the discriminandum was too large. We investigated this possibility in Protocol 2 by reducing the distance between the landmark and the discriminandum. We also simplified the spatial configuration between the discriminandum and the landmark in Protocol 2 by always placing the landmark to the right of the discriminandum, whereas in Protocol 1, the landmark was always placed medially in relation to the discriminandum (i.e., to the left of the right discriminandum and to the right of the left discriminandum).

\section{PROTOCOL 2}

The materials and procedures used in Protocol 2 were identical to those used in Protocol 1, except that the landmark was positioned so that it was in direct contact with the rewarded discriminandum (Fig. 2). Once again, dogs failed to achieve the criterion level of performance over 400 trials. The combined data from Protocols 1 and 2 suggest that the dogs are not attending to the spatial relationship between the landmark and the discriminandum. We developed Protocol 3 to determine if dogs could learn the spatial configuration between the landmark and the discriminandum if the animals were first trained to specifically attend to the landmark.

PROTOCOL 3

The third protocol had two separate stages, 1 and 2 .

$$
\begin{array}{llllllllllllllll}
L & E & A & R & N & I & N & G & \boldsymbol{\bigotimes} \\
\mathbf{5 6} & M & E & M & O & R & Y
\end{array}
$$



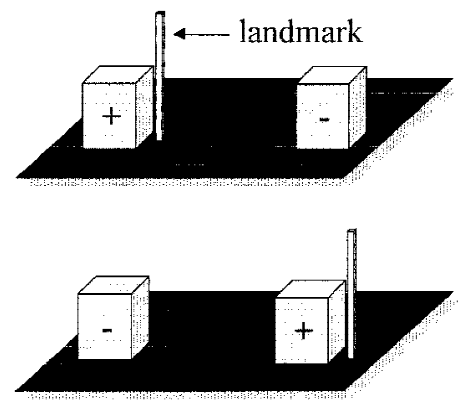

Figure 2: Landmark and discriminanda configuration for Protocol 2 in Experiment 1. The materials were identical to those used in Protocol 1 (see Fig. 1), except that the landmark was positioned so that it was in direct contact with and to the right of the rewarded discriminandum.

STAGE 1

In this task, two opaque Petri dishes were used as the identical discriminanda. They were placed $23 \mathrm{~cm}$ apart over the food wells. Velcro tabs were glued to the top center of the Petri dishes to hold the landmark in place (Fig. 3, top). This landmark position was called landmark 0 (LO). Dogs were required to associate the landmark on top of the discriminandum with the food reward. The procedure was identical to Protocols 1 and 2. Dogs were tested to criterion or 40 test sessions, whichever came first. Table 1 (first two columns on the left) shows that all dogs reached criterion in less than 40 test sessions, which suggests that dogs could be trained to attend to the landmark.

STAGE 2

Once the dogs had learned to attend to the landmark in stage 1 , we investigated whether the dogs could learn the spatial elements in the landmark configuration if the distance between the landmark and the discriminandum was now increased incrementally. The landmark was placed diagonally and medially at either $1 \mathrm{~cm}$ (landmark 1 ; L1), $2 \mathrm{~cm}$ (landmark 2; L2), $3 \mathrm{~cm}$ (landmark 3; L3), or $4 \mathrm{~cm}$ (landmark 4 ; L4) away from the discriminandum that covered the reward (Fig. 3, bottom). The procedure was identical to stage 1 . Dogs were tested at each distance (beginning with $1 \mathrm{~cm}$ ) until they either reached criterion or had received 40 test sessions. Once they had reached criterion at a given distance, they were then tested on the next distance. Dogs that did not reach criterion at a particular distance were not tested at the next dis- tance. Table 1 shows that all dogs, except two aged dogs (Millie and Inu), reached criterion at L1 in $<400$ trials. The remaining seven dogs reached criterion at L2 and L3 in 400 trials. At L4, however, only five of the seven dogs (Angus, Benz, Bronco, Santa, and Nickie) reached criterion in $<400$ trials. A one-way ANOVA was conducted to evaluate the total number of errors during acquisition of LO, L1, L2, L3, and L4 for the five dogs who successfully reached criterion at $\mathrm{L} 3$. There was a significant main effect: $F(4,24)=3.27, P<0.028$, which suggests that performance decreases as a function of increasing distance. These findings suggest that (1) dogs could learn to attend to the spatial elements of the landmark configuration task once they had learned to first attend to the landmark; and (2) the task becomes more difficult for the dogs as the distance between the landmark and the discriminandum increases. To further investigate whether task difficulty increases as a function of the distance of the landmark from the rewarded discriminandum, we tested a subset of six dogs (Angus, Benz, Bronco, Buick, Quincy, Santa) a variable distance landmark task in Experiment 2.

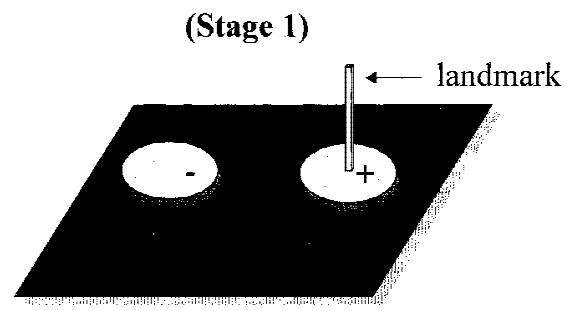

(Stage 2)

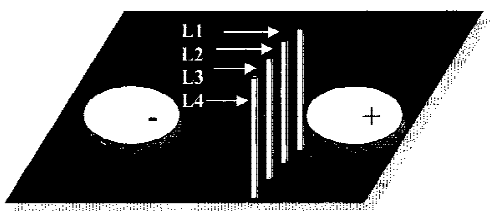

Figure 3: (Top) Landmark and discriminanda configuration for Protocol 3 (stage 1) in Experiment 1. Two opaque Petri dishes were used as the identical discriminanda and Velcro tabs were glued to the top center of the Petri dishes to hold the landmark in place. This landmark position was called landmark 0 (LO). (Bottom) Landmark and discriminanda configuration for Protocol 3 (stage 2) in Experiment 1. The materials were identical to those used in stage 1 (see $A$ ) except that the landmark was placed diagonally at either $1 \mathrm{~cm}$ (landmark $1 ; \mathrm{L} 1), 2$ $\mathrm{cm}$ (landmark 2; L2), $3 \mathrm{~cm}$ (landmark 3; L3), or $4 \mathrm{~cm}$ (landmark 4 ; L4) away from the discriminandum that covered the reward.

$$
\begin{array}{llllllllllllllll}
L & E & A & R & N & I & N & G & \boldsymbol{Q} \\
\mathbf{5 7} & M & E & M & O & R & Y
\end{array}
$$


Table 1: Acquisition of landmark discrimination task

\begin{tabular}{|c|c|c|c|c|c|c|c|c|c|c|}
\hline \multirow{2}{*}{$\begin{array}{l}\text { Dog's } \\
\text { name }\end{array}$} & \multicolumn{2}{|c|}{ LO } & \multicolumn{2}{|c|}{ L1 } & \multicolumn{2}{|c|}{ L2 } & \multicolumn{2}{|c|}{ L3 } & \multicolumn{2}{|c|}{ L4 } \\
\hline & error & trial & error & trial & error & trial & error & trial & error & trial \\
\hline Angus & 10 & 30 & 20 & 70 & 2 & 10 & 13 & 60 & 1 & 20 \\
\hline Benz & 22 & 70 & 70 & 200 & 1 & 20 & 1 & 10 & 6 & 20 \\
\hline Bronco & 24 & 50 & 1 & 10 & 4 & 209 & 32 & 80 & 151 & 400 \\
\hline Buick & 12 & 40 & 6 & 20 & 57 & 180 & 169 & 400 & 200 & 400 \\
\hline Quincy & 36 & 90 & 18 & 70 & 33 & 100 & 146 & 400 & 200 & 400 \\
\hline Santa & 17 & 50 & 1 & 10 & 6 & 30 & 18 & 60 & 34 & 100 \\
\hline Nickie & 44 & 160 & 28 & 100 & 34 & 110 & 14 & 50 & 53 & 150 \\
\hline Inu & 153 & 330 & 165 & 400 & N.A. & N.A. & N.A. & N.A. & N.A. & N.A. \\
\hline Millie & 75 & 210 & 168 & 400 & N.A. & N.A. & N.A. & N.A. & N.A. & N.A. \\
\hline
\end{tabular}

Total number of errors and total number of trials during the acquisition of L0, L1, L2, L3, and L4. Maximum number of trials performed at any landmark position is 400 (10 trials per day for a maximum of 40 days). (L0) Landmark 0 (on top of rewarded discriminandum); (L1) landmark 1 (1 cm away from rewarded discriminandum); (L2) landmark 2 (2 cm away from rewarded discriminandum); (L3) landmark 3 (3 cm away from rewarded discriminandum); (L4) landmark 4 (4 cm away from rewarded discriminandum). (N.A.) Not available.

\section{Experiment 2: Performance on a Variable Distance Landmark Task}

The purpose of Experiment 2 was to investigate whether dogs could perform the landmark task if the distance between the landmark and the rewarded discriminandum was varied across trials. The materials and procedure were identical to Protocol 1 , stages 1 and 2 . The landmark was randomly positioned at either L0, L2, or L4, as determined by the computer. Dogs were tested once a day with 10 trials per day, for a total of 40 days (400 trials). Dogs were required to associate the reward with the discriminandum that was closest to the landmark.

\section{RESULTS FOR VARIABLE DISTANCE LANDMARK DISCRIMINATION TASK}

An analysis of the total number of errors made over 400 trials using the binomial probability distribution showed that all dogs performed this variable distance landmark discrimination task at significantly greater than chance levels $(P<0.05)$. Figure 4 shows the mean percentage of correct responses as a function of the distance of the landmark from the rewarded discriminandum for all dogs. A one-way repeated measures ANOVA revealed a significant effect for distance: $F(2,10)=50.17, P<0.0021$, which shows that task performance decreases as the distance between the landmark and the rewarded discriminandum increases.

\section{Experiment 3: Performance on a Spatial Delayed Nonmatching to Position Task}

Experiment 3 was designed to determine whether dogs could also perform a spatial DNMP task, which relies on egocentric spatial cues. The subjects consisted of a subset of five dogs (Angus, Benz, Bronco, Buick, Quincy) from the previous experiment. Each of these dogs had performed

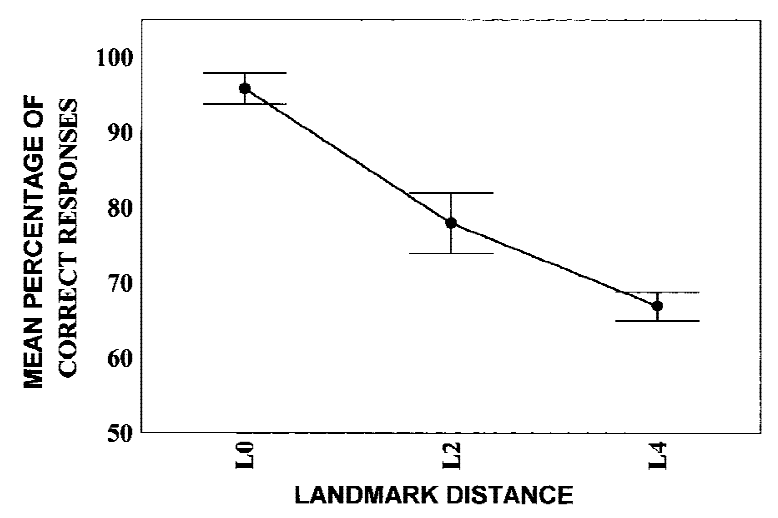

Figure 4: Mean percentage of correct responses as a function of the distance of the landmark from the rewarded discriminandum for all dogs on the variable distance landmark task in Experiment 2. A one-way repeated measures ANOVA revealed a significant effect for distance: $F(2,10)=50.17, P<0.0021$, which shows that task performance decreases as the distance between the landmark and the rewarded discriminandum increases. Values represent mean \pm S.E.M.

$$
\begin{array}{llllllllllllllll}
L & E & A & R & N & I & N & G & \boldsymbol{\bigotimes} \\
\mathbf{5 8} & M & E & M & O & R & Y
\end{array}
$$


successfully on the variable distance landmark task. The spatial DNMP task has been described previously by Head et al. (1995). Briefly, in the testing box, dogs are presented with a single object (sample) covering either the left or the right food well on the presentation tray. Following a delay, dogs are presented with two identical objects covering both the left and right food wells. To obtain the food reward, dogs are required to choose the object on the side opposite to the side where the sample was originally presented. Dogs are tested using a variable delay of 20,70 , or $110 \mathrm{sec}$ between presentation of the sample and presentation of the identical objects. All dogs had learned previously to successfully perform the DNMP task at delays of 10,20 , and 30 sec before being tested on the variable delay procedure.

\section{RESULTS FOR VARIABLE DELAY SPATIAL} DNMP TASK

An analysis of the total number of errors made across 400 trials using the binomial probability distribution showed that all dogs performed this variable delay spatial DNMP task at significantly greater than chance levels $(P<0.05)$. Figure 5 shows the mean percentage of correct responses as a function of delay for all dogs. A one-way repeated measures ANOVA revealed a significant effect for delay: $F(2,8)=6.72, P<0.019$, suggesting that task performance decreased as the delay interval increased. These findings suggest that, in addition to learning

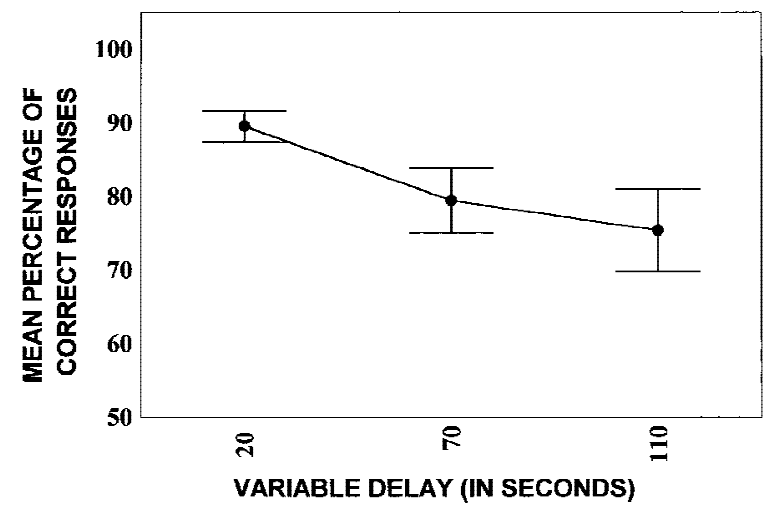

Figure 5: Mean percentage of correct responses as a function of delay for all dogs for the spatial DNMP task in Experiment 3. A one-way repeated measures ANOVA revealed a significant effect for delay: $F(2,8)=6.72$, $P<0.019$, suggesting that task performance decreased as the delay interval increased. Values represent mean \pm S.E.M. an allocentric spatial memory task (variable delay landmark discrimination task), these same dogs can also perform an egocentric spatial memory task (variable delay DNMP task).

\section{Discussion}

This series of experiments showed that (1) dogs can perform a landmark discrimination task if they are first trained to attend to the landmark; (2) dogs can use allocentric cues to solve a spatial task; and (3) dogs can perform both egocentric and allocentric spatial memory tasks.

The primary purpose of this study was to develop a protocol for testing allocentric spatial learning in dogs. We originally thought that this would not be difficult. Our initial attempts to train dogs on the landmark discrimination task (i.e., Protocols 1 and 2), however, were unsuccessful. In Protocols 1 and 2, the landmark and the rewarded discriminandum were spatially separated. Protocol 3 , however, showed that dogs could learn the spatial relationship between the landmark and the rewarded discriminandum if they were first taught to attend to the landmark, which was accomplished by placing the landmark directly on top of the rewarded discriminandum. These findings suggest that dogs were unable to learn the landmark task in Protocols 1 and 2 because they failed to attend to the relevant cue. This probably occurred because the landmark was remote from the food well. When the landmark itself became part of the discriminandum covering the food well in Protocol 3, however, subsequent acquisition of the task became relatively easy.

The apparent necessity of initially training dogs to respond to the landmark on top of the discriminandum in the LO condition, raises a potential question about whether dogs were actually learning to use allocentric cues in the subsequent testing. Because the combination of the landmark and discriminadum probably consists of a distinct object, the L0 condition is an object discrimination task (i.e., dogs may respond to the combination of the landmark and the discriminandum). Given this assumption, we cannot rule out the possibility that the animals solved the subsequent conditions (i.e., L1, L2, L3, L4) through stimulus generalization.

On the other hand, the actual response of the animals in conditions L1, L2, L3, and L4 was to directed towards the rewarded discriminandum, not to the landmark itself. This suggests that dogs were attending to the spatial relationship between

$$
\begin{array}{lllllllllllllll}
L & E & A & R & N & I & N & G & \begin{array}{l}
\boldsymbol{Q} \\
\mathbf{5 9}
\end{array} & M & E & M & O & R & Y
\end{array}
$$


the landmark and the discriminandum, and that they were not simply responding to the combination of the landmark and the discriminandum. In addition, the discriminandum and the landmark were presented to the dog on both the left and the right sides of the tray (see Fig. 3B). The configuration of the landmark and the rewarded discriminandum on the left side was, therefore, the mirror image of the configuration of the landmark and the rewarded discriminandum on the right side. The fact that the configurations were actually different, further increases the likelihood that the animals using allocentric spatial relations rather than stimulus generalization to solve the task..

Difficulty in the initial training on the landmark discrimination paradigm was also reported by Biegler and Morris (1996) in rats. Why should this seemingly simple task be so difficult? In nature, external landmarks are frequently likely to be the only available cues for spatial navigation, and the use of such external landmarks should, therefore, be relatively simple. As noted earlier, we have identified one possible reason, that of the proximity of the landmark to the food. Another important variable is landmark stability. In natural environments, landmarks are useful because they are part of a larger environmental context and are fixed in space. In the present task, however, the position of the landmark varied from trial to trial (i.e., the landmark was not stable), which may have made the task much more difficult for dogs to solve. Biegler and Morris (1996) have noted similar findings in landmark discrimination tasks in rats. Although dogs could not learn the original version of the landmark discrimination task (Protocol 1), which was based on the task used by Pohl (1973) with monkeys, it is difficult to draw specific conclusions regarding the allocentric spatial capabilities between the two species because of differences in testing procedures. For example, the correction procedures were markedly different. In the Pohl (1973) study, every incorrect response was followed by a forced-choice correction trial in which the incorrect discriminandum was removed while the correct discriminandum was left in place until the monkey made the correct choice. Compared to the one-trial correction procedure used in the present study, it is very likely that Pohl's correction procedure significantly facilitated the task learning.

We also found that performance on the landmark task decreased as a function of the distance between the landmark and the rewarded discriminandum. Dogs made significantly more errors when the distance between the landmark and the rewarded discriminandum was increased. This result is not too surprising, because previous research with rats has also found that task difficulty increases as a function of the distance between the landmark and the discriminandum (Biegler and Morris 1996). We did find, however, that dogs were able to learn a variable distance landmark task. This shows that dogs were not only able to learn the association between the reward and the object that was closest to the discriminandum, but they were also able to apply this rule when the distance between the landmark and the discriminandum were varied.

Despite the fact that most dogs learned the landmark task with little difficulty, two dogs failed to acquire the task when the landmark was placed at a distance of $1 \mathrm{~cm}$ away from the discriminandum. Both of these dogs were over 10 years of age, however, which raises the possibility that this form of allocentric spatial learning may be sensitive to age effects in the canine. Past research from our laboratory has demonstrated that aged dogs, like other species, show age-dependent deterioration in cognitive function. For example, aged dogs acquire nonspatial visual tasks (Milgram et al. 1994) and the DNMP task (Head et al. 1995) more slowly than young dogs. Because only two aged dogs were included in this study, however, we are unable to determine whether performance on the landmark discrimination task deteriorates as a function of age. Studies are currently being conducted in our laboratory to investigate this possibility.

As dogs could perform both the variable delay DNMP task and the variable distance landmark discrimination task, this suggests that dogs could learn both egocentric and allocentric spatial tasks. It is not clear from this study, however, which neural substrates are responsible for egocentric and allocentric spatial learning in the dog. As described earlier, Pohl (1973) demonstrated that lesions to the posterior parietal cortex (but not the frontal cortex) impair performance on the landmark task, whereas lesions to the frontal cortex (but not the posterior parietal cortex) impair performance on a place discrimination task that relies on egocentric cues. Based on these data, Pohl (1973) suggested that the parietal cortex may mediate allocentric spatial learning, whereas the frontal cortex may mediate egocentric spatial learning. Although the neural substrates responsible for allocentric and egocentric spatial learning in the present study are not completely understood, it is

$$
\begin{array}{lllllllllllllll}
L & E & A & R & N & I & N & G & \begin{array}{l}
\boldsymbol{Q} \\
60
\end{array} & M & E & M & O & R & Y
\end{array}
$$


entirely possible that dual neural mechanisms for spatial orientation may also exist in the canine brain.

In summary, we developed a protocol to successfully evaluate landmark discrimination learning in the dog. This study provides the first evidence that dogs can use allocentric, as well as egocentric cues, to solve a spatial learning tasks.

\section{Acknowledgments}

This research was made possible by the National Science and Engineering Research Council of Canada (grant OPG 7659) and the National Institute on Aging (grant AGI 12694).

The publication costs of this article were defrayed in part by payment of page charges. This article must therefore be hereby marked "advertisement" in accordance with 18 USC section 1734 solely to indicate this fact.

\section{References}

Biegler R. and R.G.M. Morris. 1996. Landmark stability: Further studies pointing to a role in spatial learning. Q. J. Exp. Psychol. 48: 307-345.

Fox, M.W. 1971. Integrative development of brain and behavior in the dog. University of Chicago Press, Chicago, IL.

Head, E., R. Mehta, J. Hartley, M. Kameka, B.J. Cummings, C.W. Cotman, W.W. Ruehl, and N.W. Milgram. 1995. Spatial learning and memory as a function of age in the dog. Behav. Neurosci. 109: 851-858.

Gallistel, C.R. 1990. The organization of learning. MIT Press, Cambridge, MA.

Gleason, T.C. and L.A. Rothblat. 1994. Landmark discrimination in the rat: A measure of allocentric spatial ability. Behav. Neurosci. 108: 206-209.

Hunter, W.S. 1913. The delayed reaction in animals and children. Behav. Monographs 1-86.

Lawicka, W. and J. Konorski. 1959. The physiological mechanism of delayed reactions. III. The effects of prefrontal ablations on delayed reactions in dogs. Acta Biol. Exp. 19: $221-231$.

Maguire E.A., R.S. Frackowiak, and C. Frith. 1996. Learning to find your way: A role for the human hippocampal formation. Proc. R. Soc. Lond. B. Biol. Sci. 263: 1745-1750.

Maguire E.A., C.D. Frith, N. Burgess, J.G. Dunnett, and J. O'Keefe. 1998. Knowing where things are: Parahippocampal involvement in encoding object locations in virtual large-scale space. J. Cog. Neurosci. 10: 61-76.

Milgram, N.W., E. Head, E. Weiner, and E. Thomas.1994. Cognitive functions and aging in the dog: Acquisition of nonspatial visual tasks. Behav. Neurosci. 108: 57-68.
Mishkin, M. and L.G. Ungerleider. 1982. Contribution of striate inputs to the visuospatial functions of parietopreoccipital cortex in monkeys. Behav. Brain Res. 6: 57-77.

Mishkin, M., M.E. Lewis, and L.G. Ungerleider.1982. Equivalence of parieto-preoccipital subareas for visuospatial ability in monkeys. Behav. Brain Res. 6: 41-55.

Pohl, W. 1973. Dissociations of spatial discrimination deficits following frontal and parietal lesions in monkeys. J. Comp. Physiol. Psych. 82: 227-239.

Stein, J.F. 1989. Representation of egocentric space in the posterior parietal cortex. Q. J. Exp. Physiol. 74: 583-606.

Ungerleider, L.G. and B.A. Brody. 1977. Extrapersonal spatial orientation: The role of posterior parietal, anterior frontal, and inferotemporal cortex. Exp. Neurol. 56: 265-280.

Received December 2, 1998; accepted in revised form February 3, 1999.

$$
\begin{array}{llllllllllllllll} 
& E & A & R & N & I & N & G & \mathbf{Z} & M & E & M & O & R & Y \\
61 &
\end{array}
$$




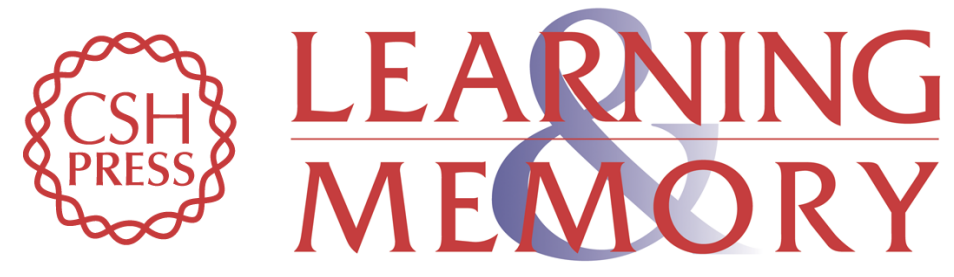

\section{Landmark Discrimination Learning in the Dog}

Norton W. Milgram, Beth Adams, Heather Callahan, et al.

Learn. Mem. 1999, 6:

Access the most recent version at doi:10.1101/Im.6.1.54

\section{License}

Email Alerting Receive free email alerts when new articles cite this article - sign up in the box at the Service top right corner of the article or click here. 